\title{
EFEITOS DA FREQÜÊNCIA DE CONHECIMENTO DE RESULTADOS NA APRENDIZAGEM DE DIFERENTES PROGRAMAS MOTORES GENERALIZADOS
}

\author{
Suzete CHIVIACOWSKY* \\ Go TANI**
}

\section{RESUMO}

O objetivo deste estudo foi verificar os efeitos da freqüência de conhecimento de resultados (CR) na aprendizagem de tarefas motoras governadas por diferentes programas motores generalizados, em um arranjo de prática randômica. Participaram do estudo 28 estudantes universitários de ambos os sexos, distribuídos em dois grupos de 14 sujeitos, de acordo com as diferentes condições de frequiência de $\mathrm{CR}$. $\mathrm{O}$ delineamento experimental abrangeu duas fases: aquisição e transferência. Os dois grupos receberam $\mathrm{CR}$ verbal e terminal. Na fase de aquisição, os grupos receberam, respectivamente, CR de 100 e $50 \%$ de frequiência relativa, num total de 120 tentativas de prática, 40 para cada tarefa, de forma randômica. Foi aplicada a análise de variância a dois fatores (grupos $\mathrm{x}$ blocos), com medidas repetidas no último fator, nas duas fases do estudo, utilizando as médias em blocos de 15 tentativas. Os resultados na fase de transferência não demostraram diferença significante entre os grupos $(p>0,05)$, permitindo concluir que a redução da freqüência relativa de $\mathrm{CR}$ não é prejudicial para a aprendizagem de movimentos pertencentes a diferentes classes ou programas motores generalizados.

UNITERMOS: Freqüência de conhecimento de resultados; Programa motor generalizado; Aprendizagem motora.

\section{INTRODUÇÃO}

Uma análise do processo evolutivo das pesquisas na área de aprendizagem motora permite identificar duas fases bastante características. A primeira é aquela que se inicia no final do século passado e se estende até os anos 70 , caracterizada por una abordagem orientada à tarefa ou ao produto. Tal abordagem procurava investigar os efeitos de diferentes variáveis como quantidade de prática, prática massificada ou distribuída, fadiga, motivação, conhecimento de resultados, entre outras, sobre a "performance" de certas tarefas motoras. Nessa abordagem, os pesquisadores não estavam preocupados com os processos ou mecanismos subjacentes à produção do movimento ou com a investigação da forma através da qual uma habilidade motora era adquirida (Kelso, 1982; Manoel, 1995; Tani, 1992).

Por volta de 1970, houve uma importante transformação ou mudança de paradigma que foi a troca da abordagem com orientação à tarefa para uma abordagem orientada ao processo (Pew, 1970), como resultado da aplicação da teoria de processamento de informações ao estudo do comportamento motor. Essa nova abordagem, que continua até o presente, procura dar ênfase às operações mentais que acontecem entre o estímulo e a resposta, ou seja, as atividades cognitivas que precedem a ação motora propriamente dita (Stelmach, 1976). Nela, o organismo humano é considerado um sistema auto-regulatório capaz de receber, processar, armazenar, transmitir e utilizar informações.

\footnotetext{
- Escola Superior de Educação Fisica da Universidade Federal de Pelotas.

**Escola de Educação Física e Esporte da Universidade de São Paulo.
} 
Com o surgimento dessa abordagem, tornou-se possível aos pesquisadores investigarem os processos ou mecanismos subjacentes que contribuem para a "performance" motora, como seleção da resposta, programação de movimentos, armazenamento de informações, detecção e correção de erros, entre outros, e não apenas os efeitos de variáveis sobre a "performance" (Adams, 1971). Nesse contexto, o fenômeno aprendizagem molora é visto como um conjunto de processos, relacionados com a prática ou experiência, que leva às mudanças relativamente permanentes na capacidade para responder (Schmidt, 1982). Tal mudança na capacidade para responder ou para executar habilidades motoras, é considerada uma decorrência de melhora nos processos ou mecanismos internos subjacentes.

Entretanto, essa busca de esclarecimento dos mecanismos e processos subjacentes levou os pesquisadores a entenderem que para se estudar como o comportamento motor muda com a prática era preciso conhecer primeiro o que muda, ou seja, o mecanismo de controle motor. Isto fez com que, nestas últimas duas décadas, os estudos sobre controle motor recebessem atenção quase que exclusiva em detrimento dos estudos em aprendizagem motora. Apenas recentemente, esse panorama tem começado a mudar. A idéia da dependência das pesquisas de aprendizagem motora aos conhecimentos de controle motor tem sido questionada com o argumento de que existem temas de investigação genuinamente pertencentes à área de aprendizagem motora quc podem ser explorados independentemente dos avanços em controle motor. Dentro dessa visão, os conhecimentos de controle motor forneceriam instrumentos teóricos para enriquecer as interpretações de dados obtidos em aprendizagem motora, mas não determinariam a viabilidade ou não de suas pesquisas (Públio, Tani \& Manoel, 1995).

Essa nova mancira de visualizar a relação entre os conhecimentos de aprendizagem e controle tcm contribuído para a retomada das pesquisas sobre a aquisição de habilidades motoras, particularmente aquelas relacionadas ao estudo das variáveis que afetam a aprendizagem. Dentre elas, conhecimento de resultados, observação e demonstração, imagem e imaginação e estrulura de prática têm recebido muita atenção dos pesquisadores (veja, por exemplo, Adams, 1990; Annett, 1985; Glencross, 1992; Lee, Swinnen \& Serrien, 1994 e Newell, 1991, para uma revisão). Os conhecimentos obtidos em controle motor têm possibilitado a realização de pesquisas em aprendizagem apoiadas numa sofisticação teórica muito maior se comparada às pesquisas na fase denominada de pesquisas orientadas à tarefa.

Uma variável considerada das mais importantes para a aprendizagem de habilidades motoras é a prática. Existem, sem dúvida, incontáveis métodos para se organizar a prática, embora seja ainda dificil compreender como estas variações afetam a aprendizagem e como interagem entre si. Diversas caracteristicas comuns das sessões de prática têm sido estudadas na literatura e a compreensão de como estas variáveis afetam a aprendizagem nos leva a um longo caminho em direção ao planejamento de práticas eficientes.

Alćm da prática $\mathrm{cm}$ si, uma outra variável de reconlıecida relevância para a aprendizagem de habilidades motoras é o conhecimento de resultados (CR). O conhecimento de resultados é uma forma de "fcedback" que informa sobre o resultado do movimento em termos do seu objetivo ambiental e possui algumas funções importantes na aprendizagem de habilidades motoras, como a motivacional (Magill, 1989; Schmidt, 1988), a de orientar o aprendiz em direção à resposta apropriada (Adams, 1971), assim como a relacional que possibilita estabelecer relações entre os comandos motores e a resposta que levam ao fortalecimento de esquemas para a produção de novos movimentos (Schmidt, 1975).

$\mathrm{O} C R$ pode ser fornecido de várias formas. Por muito tempo acreditou-se que quanto mais freqüentes, mais precisos e mais imediatos, os seus efeitos sobre a aprendizagem de habilidades motoras seriam mais patentes (Adams, 1971; Bilodeau \& Bilodeau, 1958; Bilodeau, Bilodeau \& Schumsky, 1959; Schmidt, 1975). No entanto, esta visão tem sido contrariada por estudos recentes. Salmoni, Schmidt, \& Walter (1984), em um artigo de revisão sobre os efeitos de CR, identificaram alguns experimentos que parecem contrariar esta visão até então predominante. Alguns estudos mostram que certas variações de CR, que atuam de forma a prejudicar o desempenho durante a fase de aquisição, manifestam um efeito benéfico em testes de relenção e transferência.

Schmidt, Young, Swinnen \& Shapiro (1989), seguindo os trabalhos pioneiros de Lavery (1962) e Lavery \& Suddon (1962), examinaram uma variável chamada CR sumário, onde o "feedback" sobre um conjunto de tentativas é fornecido após o término da última tentativa do conjunto. Os resultados mostraram que aumentando-se o número de tentativas do conjunto, ou seja, diminuindo-se a freqüência com que o CR é fornecido, durante a fase de aquisição, uma piora no desempenlo era observada. Entretanto, tais condições produziam um melhor resultado quando o desempenho erä medido em testes de retenção e de 
transferência. Também o estudo de Young \& Schmidt (1990), sobre o efeito do "feedback" médio, onde o aprendiz aguardava diversas tentativas antes de receber o $\mathrm{CR}$, como no $\mathrm{CR}$ sumário, mas recebia o escore médio destas tentativas, mostrou que tal tipo de arranjo é melhor para aprendizagem do que fornecer $\mathrm{CR}$ após cada tentativa. Ainda, trabalhos como os de Lee \& Carnahan (1990) e Sherwood (1988) que utilizaram uma variação de CR chamada faixa de amplitude de "feedback" (Schmidt, 1993), onde é fornecida apenas a informação de erros que excedam uma faixa pré-determinada, por exemplo, $10 \%$ de desvio do objetivo da tarefa, mostraram resultados melhores na retenção e transferência do que o CR após cada tentativa.

Outra variável que tem sido estudada neste contexto é a frequiência de $\mathrm{CR}$. Frequiência de $\mathrm{CR}$ refere-se ao número de CRs fornecidos em uma seqüência de tentativas, em relação ao número total de tentativas executadas. São distinguidas duas medidas diferentes de frequiência: a absoluta e a relativa. Frequiência absoluta de $C R$ é o número total de CRs fornecidos durante a prática. Se 80 tentativas de prática são executadas e o sujeito recebe $\mathrm{CR}$ em metade das tentativas, então a freqüência absoluta é 40 . Frequiência relativa de $\mathrm{CR}$ refere-se à percentagem de tentativas em que o $\mathrm{CR}$ é provido. É o número de $\mathrm{CRs}$ dividido pelo total de tentativas, multiplicado por 100 . No exemplo anterior, $50 \%$. De acordo com as proposições iniciais sobre os efeitos do $\mathrm{CR}$, um arranjo com uma frequiência relativa de $50 \%$ seria menos eficiente que um com $100 \%$. As tentativas sem CR não possuiriam nenhum valor, e a variável frequêência absoluta de $\mathrm{CR}$ deveria ser determinante da quantidade de aprendizagem, enquanto a freqüência relativa não teria importância se a freqüência absoluta fosse controlada.

Bilodeau \& Bilodeau (1958) foram os primeiros a investigar mais diretamente a influência das freqüências absoluta e relativa sobre a aprendizagem. Utilizando como tarefa o deslocamento de uma manivela até uma posiçĩo considerada correta, eles mantiveram a frequiência absoluta constante (10 CRs), modificando a freqüência relativa $(100,33,25$ e $10 \%)$, consequentemente, diferenciando o número de tentativas de prática $(10,30,40$ e 100 tentativas). Consideradas somente as tentativas com CR nos quatro grupos, os resultados mostraram que a quantidade de erros em cada tentativa, assim como o padrão de mudança dos erros, com a progressão das tentativas, foram praticamente os mesmos para os quatro grupos. A conclusão dos autores foi que as tentativas sem $\mathrm{CR}$ não tiveram importância $\mathrm{e}$, assim, somente a freqüência absoluta seria relevante para a aprendizagem.

Trabalhos como o de Bilodeau \& Bilodeau (1958) têm sido criticados recentemente por não ter utilizado um delineamento de transferência ou teste de retenção para separar os efeitos passageiros de "performance" (fase de aquisição) dos efeitos mais permanentes de aprendizagem (fase de transferência). Alguns estudos (Baird \& Hughes, 1972; Castro, 1988; Chiviacowsky, 1994; Chiviacowsky \& Tani ,1993; Ho \& Shea, 1978; Taylor \& Noble, 1962; Teixeira, 1993; Winstein \& Schmidt, 1990; Wulf \& Schmidt, 1989) utilizando testes de retenção e ou transferência, onde os efeitos temporários da fase de aquisição já desapareceram, têm encontrado resultados contrários aos que eram tradicionalmente enfatizados, ou seja, freqüências menores de CR são melhores para aprendizagem. Tais estudos usaram um delineamento similar ao de Bilodeau \& Bilodeau (1958), mas com teste de transferência. Os resultados contrários às conclusões anteriores, mostraram que a freqüência relativa era uma importante variável de aprendizagem, pois a diminuição da frequiência relativa ajuda a retenção de longo termo. Assim, as tentativas sem CR, fornecidas num arranjo de prática com freqüência menor do que $100 \%$, podem ter somado algo ao processo de aprendizagem, contrário à visão antiga de que tais tentativas eram essencialmente neutras.

Isto certamente contradiz as conclusões anteriores de que prover mais CR é melhor para a aprendizagem. Nesta nova visão, um número suficiente de $\mathrm{CR}$ é considerado necessário para que a "performance" ocorra, mas se a quantidade de CR for excessiva, ele se torna prejudicial à aprendizagem. Esses estudos têm mostrado que algumas condições ou variações de CR que prejudicam o desempenho durante a fase de aquisição parecem, contrariamente, melhorar o desempenho quando medido na fase de retençĩo ou transferência cm que o $\mathrm{CR}$ era retirado. Os estudos já realizados dão suporte à hipótese de orientação apresentada por Salmoni et alii (1984), na qual uma forte função orientacional ou informacional do $\mathrm{CR}$ interfere no processo de aprendizagem, fazendo com que o aprendiz produza uma dependência demasiada à esta informação, dei.ando de processar informações intrinsecas importantes para o desenvolvimento da sua capacidade de deteç̧ão e correção de erros.

Paralelamente às pesquisas sobre conhecimento de resultados, tem havido, na última década, uma considerável êllase à idéia de que a aprendizagem motora envolve a aquisição de um programa motor generalizado (PMG) para uma classe de movimentos (Schmidt, 1988). O PMG é considerado uma representação na memória para uma classe de movimentos. Quando aspectos como "timing" relativo e força 
relativa mantêm-se invariantes, os movimentos são considerados como pertencentes a uma mesma classe e governados pelo mesmo PMG. Aspectos variantes como tempo total e força total são considerados parâmetros que são adicionados ao PMG.

Um estudo que procurou verificar o efeito da frequiência de CR na aprendizagem do PMG (Wulf \& Sclmidt, 1989), utilizou três versões de uma tarefa dentro de um mesmo programa motor generalizado. Especificamente, os autores procuraram verificar se uma frequiência relativa reduzida de $\mathrm{CR}$, que tem provado melhorar o desempenho em lestes de transferência na aprendizagem de movimentos simples, também possui os mesmos efeitos na aquisição de classes de movimentos representadas pelo PMG. Neste estudo, ambos os grupos realizaram o mesmo número de tentativas de prática durante a fase de aquisição, em um arranjo de prática em blocos de seis tentativas, de modo seriado, num total de 108 tentativas para ambos os grupos. O grupo que praticou com $67 \%$ de frequiência relativa de CR obteve melhores resultados na fase de transferência que o grupo que praticou com $100 \%$ de CR. Wulf, Lee \& Schmidt (1994) taírbém procuraram verificar os efeitos da freqüência reduzida de $C R$ na aprendizagem do PMG. Neste estudo, os grupos receberam freqüências de 50 e $100 \%$ de CR durante as tentativas de prática, organizadas de uma forma seriada, e os resultados nos testes de retenção e de transferência mostraram que 0 grupo que praticou com 50\% de CR demonstrou uma aprendizagem mais efetiva do PMG que o grupo que praticou com $100 \%$ de CR. Estes resultados corroboraram os estudos anteriores sobre os efeitos da freqüência reduzida de $\mathrm{CR}$ sobre a aprendizagem de movimentos simples.

Entretanto, os efeitos da freqüência reduzida de CR ainda não foram amplamente observados na aprendizagem de movimentos governados por diferentes PMGs. Um tópico de pesquisa que tem tratado daraprendizagem de movimentos governados por diferentes PMGs está relacionado ao efeito da interferência contextual, comparando-se o arranjo de prática utilizado, randômico ou por blocos. Vários estudos têm observado uma maior eficiência da prática randômica em relação à prática por blocos para movimentos que utilizem diferentes programas motores.

O termo interferência contextual pode ser definido, segundo Magill \& Hall (1990), como o grau de interferência funcional encontrado em uma situação de prática onde várias tarefas devem ser aprendidas e praticadas em conjunto. $O$ estudo sobre os efeitos da interferência contextual tem suas origens no trabalho de aprendizagem verbal de Battig $(1966,1979)$ e a sua originalidade está na oposição à visão prevalecente na época, de que a interferência ocasionava uma transferência negativa.

O primeiro estudo sobre os efeitos da interferência contextual em aprendizagem motora foi realizado por Shea \& Morgan (1979). Basicamente, dois grupos de sujeitos praticaram três tarefas de movimentos de braço, rápidos e seqüenciais, sob condições de prática randômica ou por blocos. Os resultados mostraram que houve uma clara vantagem na "performance" dos sujeitos sob condições por blocos durante a fase de aquisição. Entretanto, quando os sujeitos foram submetidos a um teste de transferência, a prática randômica mostrou-se mais eficiente. Outros estudos como os de Del Rey, Wughalter \& Whitehurst (1982), Gabriele, Hall \& Buckolz (1987), Sekiya, Magill, Sidaway \& Anderson (1994), Ugrinowitsch \& Manoel, 1996; Wulf \& Lee (1993), os quais utilizaram tarefas basicamente de laboratório, assim como os de Goode \& Magill (1986), Wrisberg \& Liu (1991) e Chiviacowsky \& Schild (não publicado), os quais utilizaram tarefas mais próximas à siluação real, obtiveram resultados semellhantes

Existem basicamente duas hipóteses explicativas para o efeito da interferência contextual: a de elaboração e a de esquecimento. A primeira hipótese, apresentada por Shea \& Morgan (1979), propõe que a prática variada de forma randômica atua diretamente na memória aliva no sentido de proporcionar processamento múltiplo que leva ao desenvolvimento de estratégias de processamento. Como diferentes tarefas são armazenadas na memória ativa, o aprendiz necessita. identificar, em cada tentativa, a representação necessária para implementar uma determinada resposta. Em outras palavras, a interferência criada na memória durante a prática resulta na melhoria no processamento elaborativo e distintivo que posteriormente facilita a retenção e a transferência.

Uma hipólese alternativa interessante para explicar estes resultados é a de esquecimento, a qual conisidera que quando o aprendiz muda da tarefa $\mathrm{A}$ para a tarefa $\mathrm{B}$, os processos que produzem a solução para a tarefa B causam o esquecimento da solução produzida na tarefa A. Quando a tarefa A é apresentada algumas tentativas depois, o aprendiz deve produzir a solução novamente; por isso a "performance" na prática é relativamente pior. Contudo, supõe-se que este processo de geração de solução seja benéfico para a aprendizagem. Por outro lado, na prática por blocos, o aprendiz lembra a solução produzida em uma determinada tentativa e a aplica na próxima tentativa, o que minimiza o número de vezes 
que precisa produzir novas soluções. Portanto, a "performance" na prática por blocos é muito boa porque a solução, uma vez gerada, é lembrada em uma série de tentativas, mas a aprendizagem é pobre porque o aprendiz não tem que produzir repetidamente a solução para a tarefa em cada tentativa. Esta hipótese explicativa apresentada por Lee \& Magill $(1983,1985)$ é conhecida como hipótese de reconstrução do plano de ação. Mais especificamente, estes autores propõem que o sujeito deve resgatar o programa motor apropriado que representa a ação e ainda adicionar os parâmetros específicos para a obtenção do objetivo da tarefa. Este processo é visto como uma construção de um plano de ação para a resposta. Quando parte dos componentes deste plano é esquecida, o aprendiz necessita reconstruir ativamente o plano quando este é requerido novamente. $O$ resultado desta prática mais "esforçada" leva a uma melhor representação da habilidade, resultando consequentcmente numa maior retenção da mesma.

Assim, o presente estudo teve como objetivo verificar se o efeito da diminuição da freqüência de conhecimento de resultados, encontrado na aprendizagem de habilidades motoras simples, assim como na aprendizagem de uma classe de movimentos pertencente a um mesmo PMG, pode também ser observado na aprendizagem de classes de movimentos pertencentes a diferentes programas motores, em um arranjo de prática randômica. Trata-se, portanto, de uma tentativa de integração de dois tópicos de pesquisa em evidência na área de aprendizagem motora, quais sejam, a freqüência de conhecimento de resultados e a interferência contextual.

\section{MÉTODO}

\section{Sujeitos}

Participaram do estudo 28 universitários, alunos do Curso de Graduação em Educação Física, de ambos os sexos, inexperientes em experimentos de aprendizagem motora. Após a obtenção do consentimento para participação no experimento, eles foram distribuídos nos dois grupos experimentais.

\section{Material e tarefas}

Foi utilizado um alvo circular de $50 \mathrm{~cm}$ de diâmetro, impresso em cartolina e afixado em uma mesa retangular, com o seu centro à uma distância de $80 \mathrm{~cm}$ de um tapume, colocado de forma a eliminar a informação visual dos sujeitos. O tapume, da mesma largura da mesa (aproximadamente $1 \mathrm{~m}$ ) e com altura de $50 \mathrm{~cm}$, foi posicionado em frente ao sujeito, de forma que um vão de $5 \mathrm{~cm}$ permanecesse em relação à superfície da mesa por onde o botão pudesse deslizar. O centro do alvo teve o valor 100 e mediu $2 \mathrm{~cm}$; os outros espaços tiveram, respectivamente, $1 \mathrm{~cm}$ a mais de raio, com valores $96,92,88$ e assim sucessivamente a cada quadro unidades até o valor zero. $O$ alvo foi dividido em quatro partes por uma marca em forma de " $x$ " para tornar possível a obtenção da direção do erro (antes/depois e esquerda/direita). Foram utilizados um taco, um palito de madeira e um botão de tamanho médio (utilizado para a prática de futebol de mesa), como implementos.

Três tarefas motoras foram escollidas, sendo todas elas executadas a partir de uma posição sentada em uma cadeira com ambos os braços apoiados sobre a mesa. A primeira envolvia a impulsão de um botão de futebol de mesa de tamanho médio, medindo aproximadamente $2 \mathrm{~cm}$ de raio e $0,5 \mathrm{~cm}$ de espessura, com o dedo indicador da mão dominante, fazendo com que o mesmo deslizasse até o alvo. $O$ movimento consistia-se, basicamente, de uma flexão seguida de extensão do dedo indicador, com a mão semi-aberta apoiada na mesa.

A scgunda tarefa envolvia a impulsão do mesmo botão, com um palito de madeira medindo aproximadamente $6 \mathrm{~cm}$ de comprimento e $0,5 \mathrm{~cm}$ de largura, com a mão dominante, para que deslizasse até - alvo. O movimento caraclerizava-se, fundamentalmente, por uma flexão seguida de extensão da articulação do punho, com o antebraço apoiado na mesa.

A terceira tarefa consistia-se da impulsão do mesmo botão utilizado nas tarefas anteriores, com um taco de madeira de aproximadamente $7 \mathrm{~cm}$ de comprimento, por $3 \mathrm{~cm}$ de largura e $2 \mathrm{~cm}$ de espessura, com a mão dominante, para fazer com que o mesmo deslizasse até o alvo. O movimento era realizado sem apoiar a mão ou o antebraço na mesa. O taco era apoiado na mesa e o sujeito, segurando-o por cima, fazia o mesmo deslizar sobre ela, impulsionando o botão, ou seja, as articulações dos dedos e do punho mantinham- 
se fixas e o movimento era realizado, basicamente, com uma flexão seguida de extensão da articulação do cotovelo.

\section{Delincamento experimental e procedimentos}

Os sujeitos foram distribuídos aleatoriamente em dois grupos de 14 sujeitos e praticaram em diferentes condições de frequiência de CR. Os dois grupos receberam CR verbal e terminal. Na fase de aquisição, um grupo recebeu $\mathrm{CR}$ em todas as tentativas e o outro grupo recebeu em metade das tentativas, de forma alternada. Os dois grupos realizaram 120 tentativas, 40 em cada tarefa, e com um arranjo de prática randômica. Foi realizado un teste de transferência 24 horas após a fase de aquisição o qual constou de 30 tentativas (10 de cada tarefa) realizadas de forma randômica, sem CR. Foi utilizado um cronômetro digital para controlar os intervalos de tempo. O intervalo inter-tentativas foi de $10 \mathrm{~s}$, com $\mathrm{CR}$ sendo fornecido aos 5 s desse intervalo.

Ao chegar ao ambiente do experimento, cada sujeito recebeu informações sobre as tarefas a serem realizadas. Foi informado que, uma vez posicionado, ele não veria o alvo e o objetivo seria conseguir acertar um maior número de vezes no centro do mesmo. Ele também foi informado que o alvo estava dividido em quatro partes chamadas de esquerda, direita, antes e depois e que receberia informações sobre 0 espaço do alvo atingido. Além disso foi instruído que após o sinal verbal "vai" deveria iniciar as tentativas.

Em cada fase do experimento foi feita uma análise descritiva dos dados composta por uma medida de tendência central, representada pela média, e uma medida de dispersão (desvio padrão). Os dados utilizados foram as pontuações obtidas nas tentativas. Foram realizadas comparações das médias em blocos de 15 tentativas, num total de oito blocos para cada grupo. Para verificar as diferenças nos grupos e blocos de tentativas e na interação entre os dois fatores na fase de aquisição e transferência, foi efeluada uma análise de variância a dois fatores com medidas repetidas no último fator. O teste de Tukey foi utilizado para detectar as eventuais diferenças especificas. As análises estatísticas foram feitas separadamente para as duas fases experimentais, adotando-se o nível de significância de $5 \%$. Os dados foram analisados através do pacote estatístico ESTAT.

\section{RESULTADOS}

\section{Fase de aquisição}

Os resultados obtidos por cada grupo durante as fases de aquisição e transferência são apresentados graficamente na FIGURA 1 . As curvas de desempenho foram traçadas em função dos blocos de tentativas, tendo como medida da variável dependente a média da pontuação obtida em cada bloco. De modo geral pode-se observar que os grupos melhoraram o desempenho no decorrer das tentativas da fase de aquisição. As médias e os desvios padrão de cada grupo nesta fase encontram-se na TABELA 1 (Al a A8). 


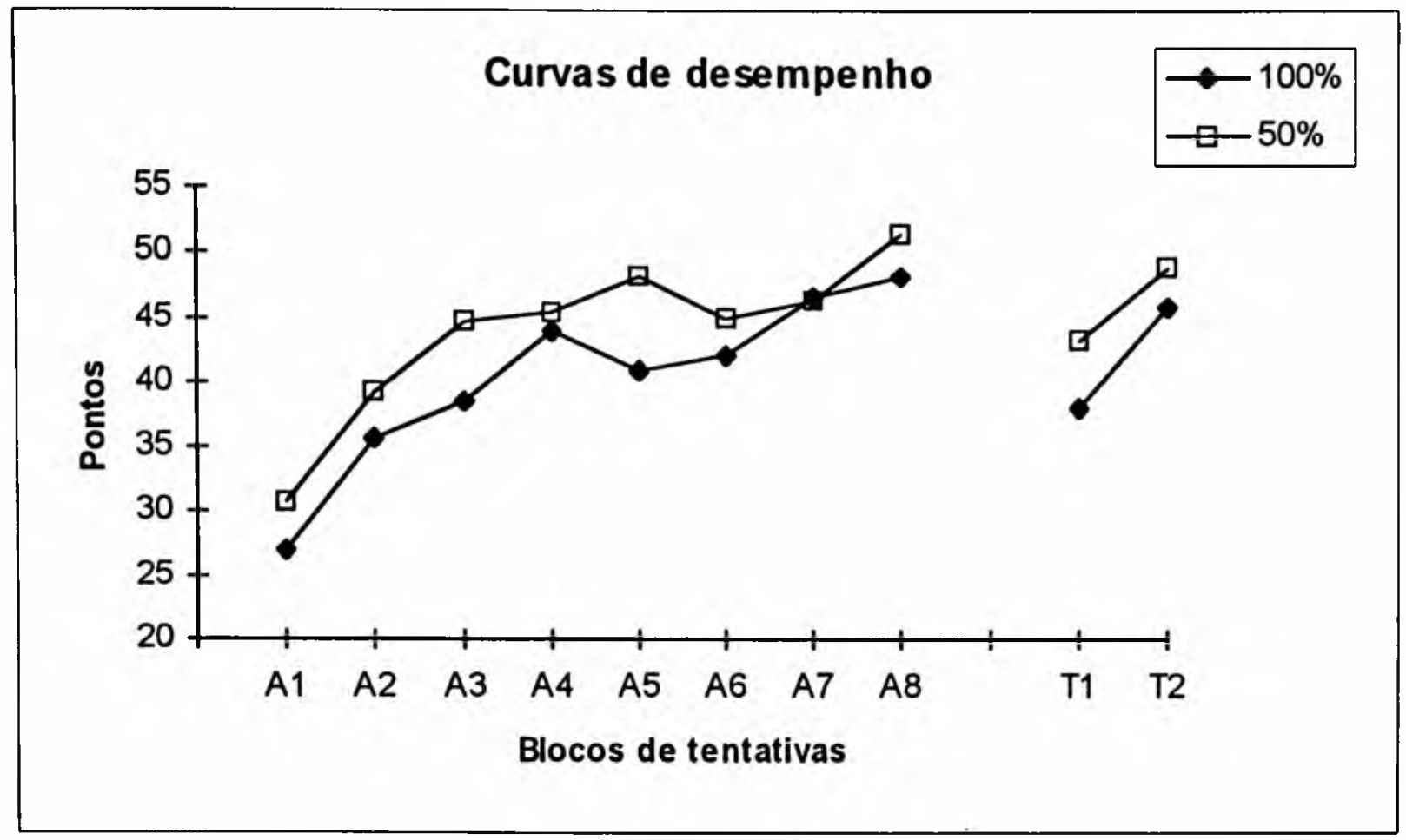

FIGURA 1 - Curvas de desempenho, nas fases de aquisição (Al a A8) e transferência (Tl e T2), dos grupos 100 e $50 \%$ de $\mathrm{CR}$, por blocos de 15 tentativas.

Analisando-se as tendências através das médias apresentadas, observou-se que o grupo $100 \%$ de CR melhorou gradativamente até o bloco 4, piorou nos blocos 5 e 6 e voltou a melhorar nos blocos 7 e 8 . Já o grupo $50 \%$ de $\mathrm{CR}$ melhorou até o bloco 5, piorou nos blocos 6 e 7 e teve seu melhor resultado no último bloco. Pode-se observar também que a pontuação obtida pelo grupo $50 \%$ de CR foi ligeiramente superior à obtida pelo grupo $100 \%$ de $\mathrm{CR}$, com exceção do bloco 7 , onde as médias praticamente não diferiram.

TABELA 1 - Médias e desvios padrão da pontuação, por bloco de tentativas, dos grupos $100 \mathrm{e}$ $50 \%$ de freqüência de $\mathrm{CR}$, nas fases de aquisição ( $\mathrm{Al}$ a $\mathrm{A8}$ ) e transferência ( $\mathrm{Tl}$ e $\mathrm{T} 2)$.

\begin{tabular}{rl|r|r|r|r|r|r|r|r|r|r}
\hline \multicolumn{2}{c|}{ Grupo } & \multicolumn{1}{|c|}{ A1 } & \multicolumn{1}{c|}{ A2 } & \multicolumn{1}{c|}{ A3 } & \multicolumn{1}{c|}{ A4 } & \multicolumn{1}{c|}{ A5 } & \multicolumn{1}{c|}{ A6 } & \multicolumn{1}{c|}{ A7 } & \multicolumn{1}{c|}{ A8 } & \multicolumn{1}{c|}{ T1 } & T2 \\
\hline $100 \%$ & X & 27,06 & 35,75 & 38,62 & 43,90 & 40,90 & 42,11 & 46,56 & 48,09 & 38,03 & 45,80 \\
& DP & 8,16 & 11,52 & 6,39 & 10,24 & 10,52 & 11,20 & 11,00 & 9,51 & 11,75 & 10,27 \\
& & & & & & & & & & \\
$50 \%$ & X & 30,78 & 39,21 & 44,60 & 45,29 & 48,08 & 44,88 & 46,37 & 51,50 & 43,17 & 49,00 \\
& DP & 8,37 & 9,94 & 10,61 & 9,43 & 8,46 & 8,48 & 9,36 & 8,27 & 9,83 & 9,72 \\
\hline
\end{tabular}

A análise de variância não revelou diferença estatisticamente significante entre os grupos $F(1 ; 26)=3,22, p=0,084$, mas sim entre os blocos $F(7 ; 182)=15,37, p=0,000$. Na interação entre blocos e grupos $F(7 ; 182)=0,51, p=0,824$, não houve diferença significante. Estes resultados mostram que os gnupos tiveram um processo de aprendizagem semelhante nesta fase, embora houvesse tendência de superioridade do grupo $50 \%$ de CR em relação ao grupo $100 \%$ de CR. Através do teste de Tukey foram realizadas comparações especificas entre os blocos. Foram detectadas diferenças entre o bloco 1 e todos os outros blocos. Também foram observadas diferenças entre o bloco 2 e os blocos 7 e 8 , e entre o bloco 3 e o bloco 8 . 
Fase de transferência

Os resultados mostram uma tendência à superioridade do grupo $50 \%$ de $\mathrm{CR}$ em relação ao grupo $100 \%$ de CR nos dois blocos. Ambos os grupos mostram resultados superiores no segundo bloco de tentativas. Através da análise de variância verificou-se, entretanto, que não houve diferença significante entre os grupos $F(1 ; 26)=1,60, p=0,217$, e na interação entre blocos e grupos $F(1 ; 26)=0,20, p=0,657$. Houve diferença significante, no entanto, entre os blocos $F(1 ; 26)=9,91, p=0,004$. Estes resultados mostram que os grupos, diferenciados pela freqüência de $\mathrm{CR}$, tiveram processo de aprendizagem semelhante, apesar de se observar uma tendência à superioridade do grupo $50 \%$ de $\mathrm{CR}$ em relação ao grupo $100 \%$ de $\mathrm{CR}$.

\section{DISCUSSÃO E CONCLUSÕES}

O objetivo do presente estudo foi verificar os efeitos da freqüência do conlecimento de resultados na aprendizagem de habilidades motoras governadas por diferentes programas motores generalizados, através da prática randômica.

Os resultados obtidos na fase de transferência mostraram uma tendência à superioridade, embora não significativa, do grupo que praticou, na fase de aquisição, com freqüência relativa de $50 \%$ de $\mathrm{CR}$ em relaçĩo ao grupo que praticou com uma frequiência relativa de $100 \%$ de CR. Estes resultados contrariam as conclusões de Adams (1971), Bilodeau (1966), Bilodeau \& Bilodeau (1958), Bilodeau et alii (1959) e Schmidt $(1975,1982)$ de que tanto maior a freqüência absoluta de $C R$, maior seria a aprendizagem. Para esses autores a aprendizagem apenas ocorre quando a informação proporcionada pelo CR pode ser utilizada para ajustar a resposta seguinte. Assim, as tentativas realizadas sem um conhecimento posterior do resultado alcançado em nada contribuiriam para melhorar a aprendizagem.

Por outro lado, os resultados encontrados estão de acordo com os resultados alcançados por Baird \& Hughes (1972), Castro (1988), Chiviacowsky \& Tani (1993), Ho \& Shea (1978), Taylor \& Noble (1962), Teixeira (1993), os quais, através de um delineamento com fase de retenção e ou transferência, mostraram que freqüências relativas menores do que $100 \%$ seriam melhores para a aprendizagem. Os resultados também corroboram os estudos de Winstein \& Schmidt (1990) e Chiviacowsky (1994), os quais mantiveram o número de tentativas constante para todos os gnupos, variando consequentemente a frequiência absoluta, e encontraram resultados melhores para freqüências menores de CR. Estão também de acordo com os trabalhos de Wulf (1992), Wulf \& Schmidt (1989) e Wulf et alii (1994), os quais mostraram que freqüências reduzidas de $\mathrm{CR}$ não prejudicam ou são até melhores para a aprendizagem de programas motores generalizados para uma mesma classe de movimentos.

O maior efeito da freqüência reduzida de CR seja talvez o desenvolvimento dos mecanismos de correção.e detecção de erros. Uma explanação interessante foi feita por Salmoni et alii (1984), relacionada à hipótese de orientação ou de dependência para o CR. Tal hipótese diz que uma menor freqüência de CR leva à aquisição de capacidade para detectar os próprios erros. Tentativas sem CR forçam o aprendiz a uma estratégia de processar seu próprio "feedback" produzido pela resposta ("feedback" intrínseco), utilizando essa informação em substituição ao CR. Tal procedimento é chamado reforço subjetivo. Ao prover CR a cada tentativa, dá-se ao aprendiz suficiente informação sobre seu progresso, reduzindo ou mesmo eliminando a necessidade de gerar informações subjetivamente. Esta capacidade de conseguir ser sensível aos próprios erros, gerada pelo reforço subjetivo, pode ser fortemente benéfica para "performance" quando CR é retirado posteriormente, fornecendo uma base para a manutenção da "performance"

Outra hipótese a ser considerada é a da consistência (Schmidt et alii, 1989), segundo a qual o $\mathrm{CR}$ após cada tentativa leva a constantes correções e, assim, os sujeitos falham em adquirir alguma consistência. Menor freqüência de CR levaria a uma maior estabilidade tentativa à tentativa, provendo uma base mais forte para o uso do CR quando este fosse apresentado. Ainda, uma outra visão sobre os efeitos da freqüência reduzida de CR foi proposta por Wulf \& Schmidt (1994), com ênfase na relação entre as informações apresentadas e a próxima ação a ser executada. Informações muito freqüentes de "feedback" podem fazer com que os processos de resgate e planejamento da próxima resposta sejam facilitados por fornecer pistas ou dicas para o aprendiz, mas isso reduz a necessidade de ou mesmo bloqueia operações consideradas importantes para a aprendizagem. 
No estudo de Wulf \& Schmidt (1994), as informações foram manipuladas de forma que o aprendiz recebesse novamente a informação de $C R$ da tentativa anterior, antes de executar a próxima tentativa. Isto numa situação de prática em blocos não provocaria nenhuma diferença, conforme observado nos resultados, pois a mesma informação seria fornecida duas vezes consecutivas em um pequeno espaço de tempo. Entretanto, numa situaçĩo de prática randômica, a informação seria repetida com um intervalo de duas ou mais tentativas em tarefas diferentes, fazendo com que as operações que o aprendiz tenha que executar para desempenhar a próxima tarefa sejam facilitadas. Com relação à prática randômica, os resultados mostraram, para a fase de aquisição, que o grupo que recebeu tais informações adicionais apresentou um desempenho pior do que o grupo que recebeu $\mathrm{CR}$ somente após cada tentativa. Os autores concluiram que tal efeito pode ter sido causado pela variabilidade nas respostas, induzida pelo "feedback" adicional, durante a prática randômica. Sobre os efeitos sobre a aprendizagem, medidos através do teste de retenção, os autores novamente não encontraram diferenças para os grupos que receberam prática por blocos. No entanto, comparando os grupos que receberam prática randômica, o grupo que recebeu informações adicionais de "feedback" teve um desempenho bem inferior ao grupo que não as recebeu. Os autores interpretaram estes resultados como um possivel bloqueio, por parte do aprendiz, das operações de resgate e planejamento, causado pelo "feedback" freqüente. Assim o "feedback" freqüente pode, além de bloquear o processamento de informações intrínsecas do movimento anteriormente executado (reforço subjetivo), bloquear os processos de resgate e construção do próximo movimento a ser executado.

Tal interpretação pode estar relacionada ìs hipóleses que tentam explicar os resultados encontrados sobre o efeito da interferência contextual (arranjo de prática randômica melhor do que em blocos para habilidades governadas por diferentes PMGs), principalmente àquela de reconstrução do plano de ação proposta por Lee \& Magill $(1983,1985)$. O plano de ação consiste em um PMG apropriado e em parâmetros que devem ser adicionados a ele. Assim, a reconstrução do plano de ação inclui os processos de construção do PMG assim como dos parâmetros necessários. Dessa forma, sob uma condição de alta interferência contextual, como no caso do arranjo de prática randômica, o aprendiz é levado a um processamento mais "esforçado" do que num arranjo de prática por blocos.

Com base nos resultados de seu estudo, Wulf \& Schmidt (1994) propuseram que o "feedback" adicional apresentado aos aprendizes pode ter eliminado os benefícios causados pela prática randômica à aprendizagem. Os autores ainda colocam a possibilidade de que os processos básicos que controlam os efeitos do "feedback" adicional e os processos que controlam os efeitos da prática em blocos sejam os mesmos, ambos bloqueando operações de resgate e construção do plano de ação. Ainda, os autores sugerem que a prática randômica pode ser mais efetiva para a aprendizagem do que a prática por blocos pela dificuldade de utilização do "feedback" quando as tarefas são apresentadas de forma randômica, ao invés de tal efetividade ser causada pelo arranjo de prálica randômica em si.

No presente estudo, a redução da frequiência de CR não foi prejudicial à aprendizagem de movimentos de classes diferentes em um arranjo de prática randômica, observando-se inclusive uma tendência de superioridade do grupo que praticou com uma frequiência de $50 \%$ comparado ao grupo que praticou com frequiência de $100 \%$. Isto pode cstar relacionado com o que ocorreu durante o intervalo pós-CR nessas duas condições experimentais. $O$ intervalo pós-CR envolve dois processos distintos. Logo após o recebimento do $\mathrm{CR}$ ocorre o relacionamento de informações obtidas via CR e via "feedback" intrínseco. Nas tentativas em que o CR não é fornecido, a avaliação é feita baseando-se apenas no "feedback" intrínseco. Num segundo momento ocorre a elaboração do plano de açĩo para a próxima tentativa.

Quando a prática é constante ou mesmo prática variada em que apenas os parâmetros são alterados, a avaliação da resposta anterior contribui diretamente com a elaboração do plano de ação da próxima tentativa. Como a revisĩo da literatura mostra, há evidências de que uma redução da freqüiência relativa de $C R$ contribui para o fortalecimento dessa capacidade de avaliação, tornando a aprendizagem mais efetiva. Em outras palavras, a elaboraçĩo de un novo plano de ação é facilitada pela capacidade melhorada de detecção e correção de erros via "feedback" intrínseco. Todavia, no caso de prática variada randômica, particularmente quando a estrutura é alterada a cada tentativa e não apenas os parâmctros, a avaliação da resposta anterior não contribui dirctamente para a elaboração do plano de ação da resposta subseqüente, por razões óbvias. Na prática variada randômica com alteração estrutural, há necessariamente uma reconstrução dos planos a cada tentativa e, portanto, a redução da frequiência relativa pode não ter cfeitos diretos na aprendizagem nessa siluação. Pode haver melhora na capacidade de deteç̧ão e correção de erros mas não na capacidade de claborar um novo plano de ação. Daí não ter havido diferença significante cntre os dois 
grupos de prática randômica com alteração estrutural do presente estudo em que a freqüência relativa de $C R$ foi alterada. É oportuno lembrar que a avaliação da resposta mostra o que está errado, mas não informa o que deve ser feilo na tentativa subseqüente. Isto depende muito mais do esforço cognitivo de reconstrução do plano de ação proporcionado, por exemplo, pela prática randômica.

Os resultados permitem concluir, portanto, que a diminuição da frequiência de CR não provoca resultados negativos para a aprendizagem de habilidades motoras como colocado anteriormente por diversos autores. Ainda, que a diminuição da freqüência de CR pode ser até benéfica, embora a superioridade da freqüência reduzida não tenha sido significante neste estudo, também para a aprendizagem de movimentos governados por diferentes programas motores generalizados, quando praticados em um arranjo de prática randômica. A forma em que tais variáveis interagem e os processos básicos que as controlam ainda não estão completamente esclarecidos, o que remete à necessidade de outros trabalhos para que conclusões mais consistentes possam ser alcançadas.

\section{ABSTRACT \\ EFFECTS OF KNOWLEDGE OF RESULTS FREQUENCY ON THE LEARNING OF DIFFERENT GENERALIZED MOTOR PROGRAMS}

The purpose of the present study was to verify the effects of frequency of knowledge of results (KR) on the learning of motor tasks governed by different generalized motor programs, using a random practice schedule. Twenty eight university students participated in the experiment, they were distributed in two groups of 14 subjects (eigth females, six males), according to different practice conditions. The experimental design encompassed two phases: acquisition and transfer. The two groups received verbal and terminal $\mathrm{KR}$. In the acquisition phase, one group practiced at a $100 \% \mathrm{KR}$ frequency and the other at a $50 \%$ KR frequency schedule. A two-tway ANOVA (groups $x$ blocks) with repeated measures on the last factor was applied in the two phases of the experiment in which the mean of 15 trials block was used. No signifficant difference between the groups was found in the transfer phase suggesting that the reduced KR frequency is not detrimental to the learning of movements governed by different generalized motor programs.

UNITERMS: Frequency of knowledge of results; Generalized motor programs; Motor learning.

\section{NOTA}

1. Esta pesquisa recebeu Bolsa de Iniciação Científica da FAPERGS, a qual foi destinada à acadêmica Cimara Correa Machado.

\section{REFERÊNCIAS BIBLIOGRÁFICAS}

ADAMS; J.A. The changing face of motor learning. Human Movement Science, v.9, p.209-20, 1990.

A closed-loop theory of motor learning. Journal of Motor Bchavior, v.3, p.111-50, 1971.

ANNETT, J. Motor learning: a review. In: HEUER, H.; KLEINBECK, U.; SCHMIDT, K.H., eds. Motor behavior: programming, control, and acquisition. New York, Springer-Verlag, 1985. p.189-212.

BAIRD, I.S.; HUGHES, G.H. Effects of frequency and specilicity of information feedback on acquisition and extinction of a positioning task. Perceptual and Motor Skills, v.34, p.567-72, 1972.

BATTIG, W.F. Facilitation and interierence. In: BILODEAU, E.A., ed. Acquisition of skill. New York, Academic Press, 1966.

The tlexibility of human memory. In: CERMAK, L.S.; CRAIK, F.I.M., eds. Levels of processing in human memory. Hillsdale, New Jersey, 1979.

BILODEAU, E.A., ed. Acquisition of skill. New York, Academic Press, 1966.

BILODEAU, E.A.; BILODEAU, I.M. Variable frequency of knowledge of results and the leaning of a simple skill. Journal of Experimental Psychology, v.55, n.4, p.379-83, 1958. 
BLODEAU, E.A.; BLODEAU, I.M.; SCHUMSKY, D.A. Some effects of introducing and withdrawing knowledge of results early and late in practice. Journal of Experimental Psychology, v.58, n.2, p.142-4, 1959.

CASTRO, I.J. Efeitos da freqüência relativa do feedback extrínseco na aprendizagem de uma habilidade motora discreta simples. São Paulo, 1988. 103p. Dissertação (Mestrado) - Escola de Educação Física, Universidade de São Paulo.

CHIVIACOWSKY, S. Frequência absoluta e relativa do conhecimento de resultados na aprendizagem de uma habilidade motora em crianças. Revista Kinesis, v.14, p.39-56, 1994.

CHIVIACOWSKY, S.; SCHILD, J.F Efeito da interferência contextual na aprendizagem de três habilidades motoras do atletismo. /não publicado/.

CHIVIACOWSKY, S.; TANI, G. Efeitos da frequiência do conhecimento de resultados na aprendizagem de uma habilidade motora em crianças. Revista Paulista de Educação Fisica, v.7, n.1, p.45-57, 1993.

DEL REY, P.; WUGHALTER, E.H.; WHITEHURST, M. The effect of contextual interference on females with varied experience in open sport skills. Research Quarterly for Exercise and Sport, v.53, n.2, p. 108-15, 1982.

GABRIELE, T.E.; HALL, C.R.; BUCKOLZ, E.E. Practice schedule effects on the acquisition and retention of a motor skill. Human Movement Science, v.6, p.1-16, 1987.

GLENCROSS, D.J. Human skill and motor learning: a critical review. Sport Science Review, v.1, n.2, p.65-78, 1992.

GOODE, S.; MAGLL, R.A. Contextual interference effects in leaning three badminton serves. Research Quarterly for Exercise and Sport, v.57, n.4, p.308-14, 1986.

HO, L.; SHEA, J.B. Effects of relative frequency of knowledge of results on retention of a motor skill. Perceptual and Motor Skills, v.46, p.859-66, 1978.

KELSO, J.A.S. Human motor behavior: an introduction. New Jersey, Lawrence Erlbaum, 1982.

LAVERY, J.J. Retention of simple motor skills as a function of type of knowledge of results. Canadian Journal of Psychology, v.16, 1.4, p.300-11, 1962.

LAVERY, J.J.; SUDDON, F.H. Retention of simple motor skills as a function of the number of trials by wich KR is delayed. Perceptual and Motor Skills, v.15, p.231-7, 1962.

LEE, T.D.; CARNAHAN, H. Bandwidth knowledge of results and motor leaning: more than just a relative frequency effect. Quarterly Journal of Experimental Psychology, v.42A, p.777-89, 1990.

LEE, T.D.; MAGILL, R.A. Can forgetting facilitate skill acquisition? In: GOODMAN, D.; WILBERG, R.B.; FRANKS, I.M., eds. Differing perspectives in motor learning, memory, and control. Austerdam, North-Holland, 1985. p.3-22.

The locus of the contextual interference in motor-skill acquisition. Journal of Experimental Psychology: Leaning, Memory and Cognition, v.9, n.4, p.730-46, 1983.

LEE, T.D.; SWINNEN, S.P.; SERRIEN, D.J. Cognitive effort and motor learning. Quest, v.46, n.3, p.328-44, 1994.

MAGILL, R.A. Motor learning: concepts and applications. 3.ed. Iowa, Wm. C. Brown, 1989.

MAGLL, R.A.; HALL, K.G. A review of the contextual interference effect in motor skill acquisition. Human Movement Science, v.9, p.241-89, 1990.

MANOEL, E.J. Aprendizagem motora: o processo de aquisição de ações motoras habilidosas. In: NETO, A.D.; GOELLNER, S.L.; BRACHT, V.L., orgs. As ciências do esporte no Brasil. Campinas, Autores Associados, 1995.

NEWELL, K.M. Motor skill acquisition. Annual Review of Psychology, v.42, p.213-37, 1991.

PEW, R.W. Toward a process-oriented theory of human skilled performance. Journal of Motor Behavior, v.2, p.8-24, 1970.

PÚBLIO, N.S.; TANI, G.; MANOEL, E.J. Efeitos da demonstração e instnıção verbal na aprendizagem de habilidades motoras da ginástica olimpica. Revista Paulista de Educação Fisica, v.9, n.2, p.111-24, 1995.

SALMONI, A.W.; SCHMIDT, R.A.; WALTER, C.B. Knowledge of results and motor learning: a review and critical reappraisal. Psychological Bulletin, v.95, n.3, p.355-86, 1984.

SCHMIDT, R.A. Aprendizagem e performance motora: dos princípios à prática. São Paulo, Movimento, 1993.

Motor control and learning: a behavioral emphasis. Champaign, Human Kinetics, 1982. 2.ed. Champaign, Human Kinetics, 1988.

A schema theory of discrete motor skill learning. Psychological Review, v.82, p.225-60, 1975.

SCIMIDT, R.A.; YOUNG, D.E.; SWINNEN, S.; SHAPIRO, D.C. Summary knowledge of results for acquisition: support lor the guidance hypothesis. Journal of Experimental Psychology: Learning, Memory and Cognition, v.15, n. 2, p. 352-59, 1989.

SEKIYA, H.; MAGLLL, R.A.; SDAWAY, B.; ANDERSON, D.I. The contextual interference effect for skill variations from the same and different generalized motor programs. Research Quarterly for Exercise and Sport, v.65, n.4, p.330-8, 1994.

SHEA, J.B.; MORGAN, R.L. Contextual interference effects on the acquisition, retention, and transfer of a motor skill. Journal of Experimental Psychology: Human Leaming and Memory, v.5, p. 179-87, 1979.

SHERWOOD, D.E. Eflects of bandwidth knowledge of results on movement consistency. Perceptual and Motor Skills, v.66, p.535-42, 1988. 
STELMACH, G.E., ed. Motor control: issues and trends. New York, Academic Press, 1976.

TANI, G. Contribuições da aprendizagem motora à educação fisica: uma análise critica. Revista Paulista de Educação Física, v.6, n.2, p.65-72, 1992.

TAYLOR, A.; NOBLE, C.E. Acquisition and extinction phenomena in human trial-and-error learning under different schedules of reinforcing feedback. Perceptual and Motor Skills, v. 15, p.31-44, 1962.

TELXEIRA, L.A. Freqüência de conlhecimento de resultados na aquisição de habilidades motoras: efeitos transitórios e de aprendizagem. Revista Paulista de Educação Física, v.7, n.2, p.8-16, 1993.

UGRINOWITSCH, H.; MANOEL, E.J. Interterência contextual: manipulação de aspecto invariável e variável. Revista Paulista de Educação Física, v. 10, n. l, p.48-58, 1996.

WINSTEIN, C.J.; SCHMIDT, R.A. Reduced frequency of knowledge of results enhances motor skill leaming. Journal of Experimental Psychology: Leaning, Memory and Cognition, v.16, 1.4, p.677-91, 1990.

WRISBERG, C.A.; LIU, Z. The effect of contextual variety on the practice, retention and transfer of an applied motor skill. Research Quarterly for Exercise and Sport, v.62, p.406-12, 1991.

WULF, G. Reducing knowledge of results can produce context effects in movements of the same class. Journal of Human Movement Studies, v.22, p.71-84, 1992.

WULF. G.; LEE, T.D. Contextual interference in movements of the same class: differential effects on program and parameter learning. Journal of Motor Bchavior, v.25, n.4, p.254-63, 1993.

WULF, G.; LEE, T.D.; SCHMIDT, R.A. Reducing knowledge of results about relative versus absolute timing: differential effects on learning. Journal of Motor Behavior, v.26, n.4, p.362-69, 1994.

WULF, G.; SCHMIDT, R.A. Feedback-induced variability and the learning of generalized motor programs. Journal of Motor Behavior, v.26, n.4, p.348-61, 1994.

The learning of generalized motor prograns: reducing the relative frequency of knowledge of results enhances memory. Journal of Experimental Psychology: Learning, Memory and Cognition 2 v. 15, n.4, p.748-57, 1989.

YOUNG, D.E.; SCHMIIT, R.A. Units of motor behavior: modifications with practice and feedback. In: JEANNEROD, M., ed. Attention and performance XIII. Hillsdale, Lawrence Erlbaum, 1990. p.763-95. 